\title{
Crystallization Kinetics of Fractionated Polyethylenes at High Pressures by a DTA Method
}

\author{
Shuichi SAWADA and Takuhei NosE \\ Department of Polymer Chemistry, Tokyo Institute of Technology, \\ Ookayama, Meguro-ku, Tokyo 152, Japan.
}

(Received October 25, 1978)

\begin{abstract}
The crystallization kinetics of fractionated polyethylenes under high pressure was studied by the DTA method for samples having molecular weights of 15500 and 150000 . In extendedchain crystallization there was a saturation phenomenon on producing extended-chain crystals. The amount of saturation was dependent on the crystallization temperature. The saturation phenomenon seems to be an important factor for considering the mechanism of growth in extended-chain crystals. The Avrami exponent of extended-chain crystals was almost two. In the case of the folded-chain crystallization, the Avrami exponent was about three, irrespective of pressure. The crystallization rate of folded-chain crystals became more rapid as pressure was increased owing to the depression of the value of excess free energy of the fold surface. The crystallization rate of extended-chain crystals is atso considered to increase with pressure by indirect comparison, and seems to have a maximum crystallization rate near the melting temperature. The estimation of lamellar thickness is discussed for folded-chain crystals produced under high pressure.

KEY WORDS Polyethylene / Crystallization Kinetics / High Pressure / Saturation Phenomenon / Avrami Exponent / Extended-Chain Crystallization / Excess Free Energy of Fold Surface /
\end{abstract}

Up to the present, many studies on crystallization under high pressure have been carried out, and it is considered that extended-chain and folded-chain crystallization are processes independent of each other. ${ }^{1-4}$ But the growth mechanism of extendedchain crystals has not been sufficiently studied. Bassett, et al., related the growth of extended-chain crystals to the appearance of the high pressure intermediate phase, that is, extended-chain crystals can be produced only via the high pressure phase. ${ }^{5}$ It is not likely, however, that the intervention of the high pressure phase is the only means for producing extended-chain crystals. ${ }^{6}$

In order to elucidate the mechanism of extendedchain crystal growth under high pressure, the studies on crystallization kinetics are essential, as well as the investigation of kinetic competition: how the crystallization process in extended-chain crystals competes with that in folded-chain crystals. Compared foldedchain crystals, however, the kinetics of extendedchain crystallization under high pressure has not been fully investigated. ${ }^{1,7,8}$
In this study, crystallization kinetics in that region where folded-chain and extended-chain crystals coexist was studied by differential thermal analysis (DTA) adopting a pressure quenching technique. This method has the advantage of being capable of separating folded-chain and extended-chain crystallization processes in isotherms and therefore makes possible the study of kinetic competition, although considerable time is necessary to carry out the experiment.

\section{EXPERIMENTAL}

The samples employed in this work were fractionated polyethylenes $\left(M_{w} / M_{n}\right.$, the ratio of the weight- to number-average molecular weight, was in the range 1.2-1.3) with molecular weights of $M_{v}=15500$ and 150000 . A DTA cell capable of measuring up to $5000 \mathrm{~kg} / \mathrm{cm}^{2}$ was designed and used for studying the melting and crystallization behavior under high pressure. The DTA cell is shown in Figure 1. 


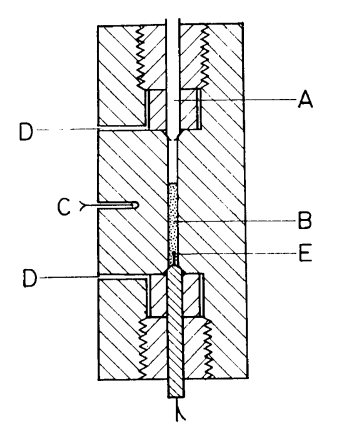

Figure 1. A DTA cell: (A) high pressure inlet; (B) sample; (C) (thermocouple) reference; (D) weep hole; (E) thermocouple.

Pressure was generated by the New High Pump (Seiritsu Kogyo Co.) and measured by the change in the electric conductivity of manganin wire, and the temperature was measured by an alumel-chromel thermocouple $(0.3 \mathrm{~mm}$ in diameter $)$. Silicone oil (Shinetsu Co. KF 96, 10 c.s.) was used as a pressure transmitting fluid. A sample of about $80 \mathrm{mg}$ was put into the cylindrical sample tube $(60 \mathrm{~mm}$ in length and $2 \mathrm{~mm}$ in diameter). The equilibrium melting temperature $T_{\mathrm{m}}{ }^{0}$ at high pressures was determined by the melting temperature of the extended-chain crystals previously produced under high pressure.

First, in order to carry out the isothermal crystallization, the materials were completely melted and held at a constant predetermined temperature within an accuracy of $\pm 0.1^{\circ} \mathrm{C}$. Then, the pressure was increased to the value required for crystallization in less than $30 \mathrm{~s}$ and kept constant within an accuracy of $\pm 10 \mathrm{~kg} / \mathrm{cm}^{2}$ for over half a day and $\pm 30 \mathrm{~kg} / \mathrm{cm}^{2}$ for over two days. The isothermal crystallization was stopped by the pressure quench conducted by raising the pressure by about $800 \mathrm{~kg} / \mathrm{cm}^{2}$ within $6 \mathrm{~s}$. Raising pressure by $1000 \mathrm{~kg} / \mathrm{cm}^{2}$ causes an increment in the melting point by about $20^{\circ} \mathrm{C}$ in the case of polyethylene. Hence the pressure quench corresponds to the cooling rate of about $160^{\circ} \mathrm{C} / \mathrm{min}$. After that the sample was cooled at a cooling rate of about $5^{\circ} \mathrm{C} / \mathrm{min}$. After the sample was cooled to room temperature, the pressure was released. The sample was then heated at a constant heating rate of $2^{\circ} \mathrm{C} / \mathrm{min}$ to give rise to endothermic traces at atmospheric pressure.

Two types of crystals (folded- and extended-chain crystals) were identified by their melting points at atmospheric pressure: the crystals with a lower

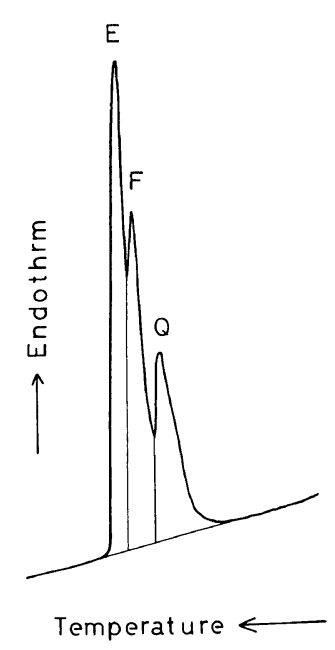

Figure 2. Schematic endothermic trace to show the way for determination of the weight fraction for the materials crystallized for a time $t$ : $\mathrm{Q}$ corresponds to the endothermic trace of crystals produced by pressure quench, $F$ to that of folded-chain crystals, and $\mathrm{E}$ to that of extendedchain crystals.

melting point of about $135^{\circ} \mathrm{C}$ were folded-chain crystals and those with a higher melting point of about $142^{\circ} \mathrm{C}$ were extended-chain crystals. When either the folded-chain or the extended-chain crystallization took place during isothermal crystallization under high pressure, each frozen sample showed two endothermic peaks in its DTA trace at atmospheric pressure: one was a trace of the crystallite produced during the isothermal crystallization and the other, a trace of that crystallized in the course of the cooling process by the pressure quench. When both the folded-chain and the extended-chain crystallizations took place, three endothermic traces were obtained, adding a third trace to these two traces.

The resultant endothermic traces were analyzed to estimate the amount of material crystallized for a time $t$, as shown in Figure 2. Each portion of the endothermic trace was estimated by weighing the copied paper of the endothermic trace. We designate $w_{1}, w_{2}$, and $w_{3}$ as the weight of the copied paper for the portion produced in the pressure quench, the weight for the folded-chain crystals, and the weight for the extended-chain crystals, respectively. The relative degree of completion of the transformation $X$ is expressed as $X=w_{2} /\left(w_{1}+w_{2}+w_{3}\right)$ for the folded-chain crystallization and $X=w_{3} /\left(w_{1}+w_{2}+\right.$ $w_{3}$ ) for the extended-chain crystallization, and if no 
extended-chain crystallization occurs, $w_{3}=0$.

The analysis of crystallization kinetics was carried out by using the Avrami equation

$$
1-X=\exp \left(-k t^{n}\right)
$$

where $k$ is the crystallization rate constant and $n$ is Avrami exponent. The crystallization rate constant $k$ was evaluated from $t_{0,1}$, the time to transform $10 \%$ of the sample.

$$
\log k=-n \log t_{0,1}+\log (\ln 10 / 9)
$$

\section{RESULTS AND DISCUSSION}

\section{Saturation Phenomenon in Extended-Chain Crystallization}

The crystallization isotherms for the samples with molecular weights 15500 and 150000 are shown in Figures 3 and 4 . For a molecular weight of 15500, the extended-chain crystals were not obtained at $P=2490 \mathrm{~kg} / \mathrm{cm}^{2}$, so that Figure $3 \mathrm{a}$ shows the crystallization isotherms of folded-chain crystals.

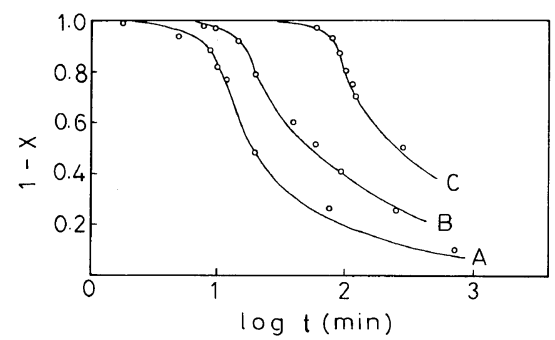

(a)

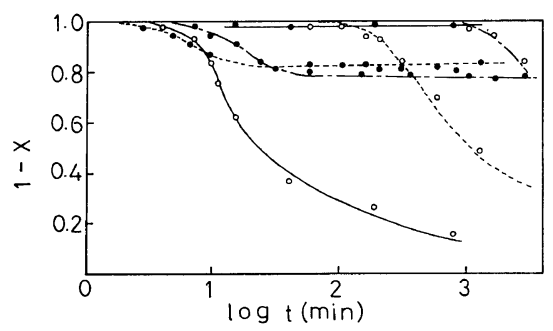

(b)

Figure 3. Crystallization isotherms for $M_{v}=15500$ : (a) pressure $P=2490 \mathrm{~kg} / \mathrm{cm}^{2}\left(T_{\mathrm{m}}{ }^{0}=197.9^{\circ} \mathrm{C}\right)$, temperature at (A) $186.6^{\circ} \mathrm{C}$, (B) $187.5^{\circ} \mathrm{C}$, and (C) $188.5^{\circ} \mathrm{C}$; (b) pressure $P=3000 \mathrm{~kg} / \mathrm{cm}^{2}\left(T_{\mathrm{m}}{ }^{0}=206.1^{\circ} \mathrm{C}\right)$, temperature at $(\square)$ $196.6^{\circ} \mathrm{C},(----) 197.8^{\circ} \mathrm{C},(--) 198.8^{\circ} \mathrm{C}$. $\bigcirc$ denotes folded-chain crystallization and denotes extendedchain crystallization.

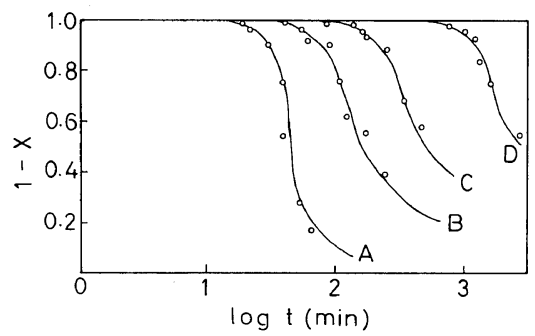

(a)

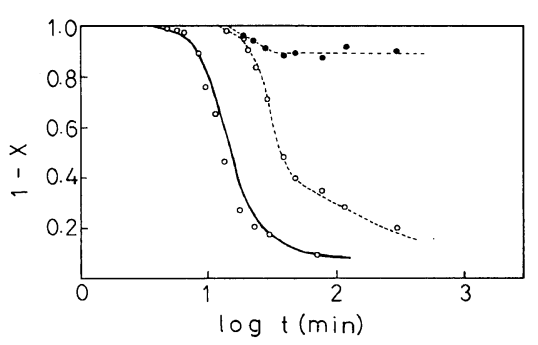

(b)

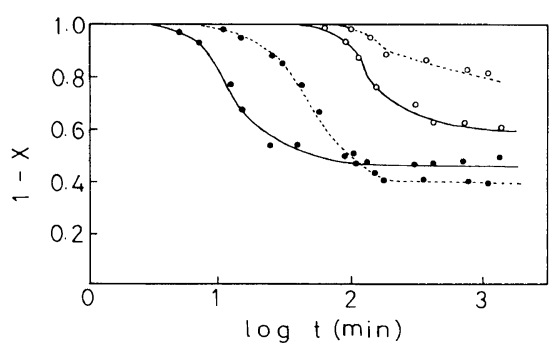

(c)

Figure 4. Crystallization isotherms for $M_{v}=150000$ : (a) pressure $P=2340 \mathrm{~kg} / \mathrm{cm}^{2}\left(T_{\mathrm{m}}{ }^{0}=196.2^{\circ} \mathrm{C}\right)$, temperature at (A) $183.6^{\circ} \mathrm{C}$, (B) $185.3^{\circ} \mathrm{C}$, (C) $186.3^{\circ} \mathrm{C}$, and (D) $187.3^{\circ} \mathrm{C}$; (b) pressure $P=2490 \mathrm{~kg} / \mathrm{cm}^{2}\left(T_{\mathrm{m}}{ }^{0}=200.1^{\circ} \mathrm{C}\right)$, temperature at (-) $187.8^{\circ} \mathrm{C}$ and (-----) $189.5^{\circ} \mathrm{C}$; (c) pressure $P=2490 \mathrm{~kg} / \mathrm{cm}^{2}$, temperature at (-) $190.5^{\circ} \mathrm{C}$ and (-- - $191.4^{\circ} \mathrm{C}$. $\bigcirc$ denotes folded-chain crystallization and denotes extended-chain crystallization.

Table I. Saturation amount $\alpha$

\begin{tabular}{cccc}
\hline \multicolumn{2}{c}{$M_{v}=15500$} & $M_{v}=150000$ \\
\cline { 3 - 4 }$T,{ }^{\circ} \mathrm{C}$ & $\alpha, \%$ & $T,{ }^{\circ} \mathrm{C}$ & $\alpha, \%$ \\
\cline { 3 - 4 } 196.6 & 1.5 & 187.8 & 0.5 \\
197.8 & 18 & 189.5 & 11 \\
198.8 & 23 & 190.5 & 53 \\
& & 191.4 & 60 \\
\hline
\end{tabular}


The production of extended-chain crystals became possible by increasing the crystallization temperature at $P=3000 \mathrm{~kg} / \mathrm{cm}^{2}$. At this pressure the induction period of extended-chain crystals was very short. However, at the later stage, no growth of extended-chain crystals occurred as shown in Figure $3 b$.

In the case of the molecular weight 150000 , at $P=2340 \mathrm{~kg} / \mathrm{cm}^{2}$ the formation of extended-chain crystals was not possible within the range of this experiment, as shown in Figure 4a. At $P=2490$ $\mathrm{kg} / \mathrm{cm}^{2}$, extended-chain crystals were formed when the temperature was high, while in the molecular weight of 15500 , no extended crystals were formed at this pressure. The formation of extended-chain crystals was possible at a lower pressure than in the case of the molecular weight of 15500 . The dependence of the formation of extended-chain crystals on molecular weight will be reported in detail in the next publication.

Figures $4 \mathrm{~b}$ and $4 \mathrm{c}$ indicate that the production of extended-chain crystals was rapid, but that the growth of these was retarded and finally saturated. This behavior is the same for extended-chain crystallization in a molecular weight of 15500 .

The amount of saturation increased as the crystallization temperature increased at constant pressure as is tabulated in Table I. The saturation phenomenon seems scarecely appear at higher crystallization temperature regions which are considered favorable from a morphological point of view for producing extended-chain crystals.

Figure 5 shows representative endothermic curves at atmospheric pressure of the materials produced during both isothermal crystallization and pressure quench. At those temperature regions where formation of extended-chain crystals was possible, the endothermic curves of DTA trace corresponding to extended-chain crystals first appeared and the endothermic area of these enlarged with crystallization time; however, after a certain crystallization time, it became difficult to produce extended-chain crystals. Folded-chain crystals also began to appear and the endothermic area of these steadily increased, in contrast to the case of growth of the extended-chain crystals.

The saturation phenomenon was reported for the so-called highly extended-chain crystallization by a dilatometric method $^{9}$ and for both growth process of high pressure phase and extended-chain crystals by
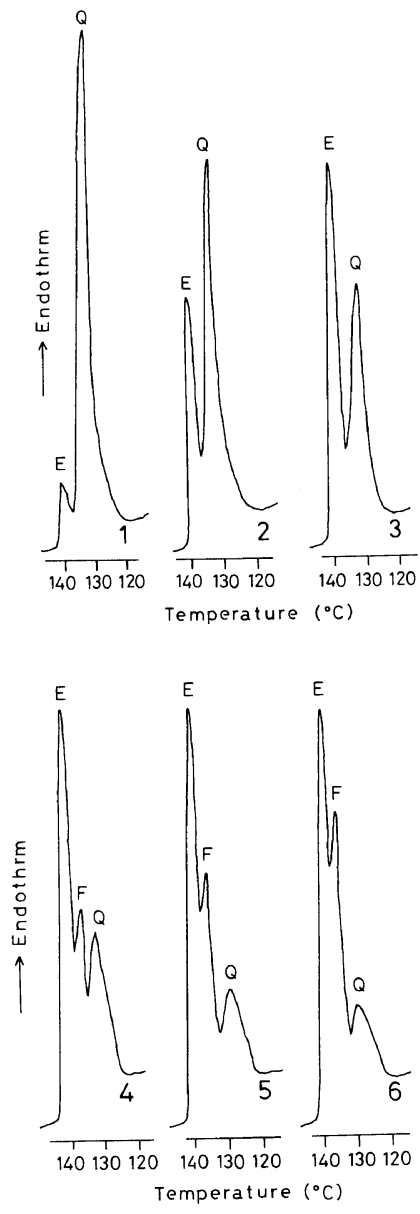

Figure 5. Change in endothermic traces with time at $P=2490 \mathrm{~kg} / \mathrm{cm}^{2}$ and $T=190.5^{\circ} \mathrm{C}$ for $M_{v}=150000$ : crystallization time (1) $7 \mathrm{~min}$, (2) $15 \mathrm{~min}$, (3) $90 \mathrm{~min}$, (4) $5 \mathrm{~h}$, (5) $12 \mathrm{~h}$, and (6) $22.5 \mathrm{~h}$. Q denotes crystallization by pressure quench, $F$ folded-chain crystallization, and $\mathrm{E}$, the extended-chain crystallization.

X-ray measurement. ${ }^{6}$ However, little attention has been paid to the saturation phenomenon.

In connection with the growth of extended-chain crystals, it was noticed that the amount of extendedchain crystals produced during isothermal crystallization was saturated. Since there has been no report of the saturation phenomenon appearing in foldedchain crystallization, it is considered that the saturation phenomenon is characteristic to the growth of extended-chain crystals.

Two aspects may be considered in regard to the saturation phenomenon. The first is that at tempera- 
ture regions where extended-chain crystals can be produced, extended-chain crystals are produced at first. But after a certain time, folded-chain crystals also begin to appear, and therefore, it becomes hard to produce further extended-chain crystals because of the rapid growth of folded-chain crystals. Secondly the extended-chain crystal growth is independent of folded-chain crystal growth; that is, the latter has little influence on the former, and the saturation phenomenon has a close connection with the crystal growth of extended-chain crystals itself. At the present, no clear conclusion can be drawn about the two aspects, but the first aspect may be negatived, judging from Figure $3 \mathrm{~b}$ which indicates that some time was necessary to initiate folded-chain crystallization following the occurrence of the saturation phenomenon.

Concerning the second aspect, we discuss briefly the saturation phenomenon in a qualitative way. It is considered that the lamellar thickness of extendedchain crystals for high molecular weight observed at atmospheric pressure is not generally coincident with the thickness of the critical nucleus. Thus, such a nucleus with a contour length of a molecule cannot be easily produced mainly because of the increase in the activation free energy of the nucleus formation. It may be assumed that the model of the bundle-like nucleus formation following the longitudinal growth of the chain is most likely. At lower crystallization temperatures, if the yielding lamellar thickness is assumed not so thick, it becomes hard to accommodate the bundles of chains emanating from the crystal in the amorphous region as crystallization proceeds. ${ }^{10}$ This results in the difficulty of incorporating additional chains in the crystal. This situation thus may lead to the saturation phenomenon.

\section{Avrami Exponent}

Table II shows the sets of the Avrami exponent $n$ estimated from the relation of the double logarithm of $(1-X)$ with $\log t$. When both the folded-chain and the extended-chain crystallization occurred, the Avrami exponent was independently determined for each crystallization isotherm. When the final amount of transformation was small, estimation of the Avrami exponent was so difficult that such data are ommited in Table II.

Table II can be divided into two groups: one for the folded-chain crystallization having an Avrami exponent of about three, and the other, an extendedchain crystallization with an Avrami exponent of about two. The exponent of two coincides with that obtained by Yasuniwa, et al. ${ }^{6}$ and Hoehn, et al. ${ }^{11}$ Kyotani and Kanetsuna obtained a slightly different exponent, ${ }^{7}$ but Hoehn, et al., recalculated the value of the Avrami exponent from the data of Kyotani and Kanetsuna and got a value of $n$ close to two.

A value of three for the folded-chain crystallization was typical for atmospheric pressure. It is thus presumed that the value of the Avrami exponent in folded-chain crystallization is little affected by pressure. On the other hand, a value of two for an extended-chain crystallization at high pressure was different from that for an extended-chain crystallization at atmospheric pressure giving a value of about four. ${ }^{12}$ This difference in Avrami exponent is considered to have some connection with the difference in the form of the extended-chain crystallization mechanism between atmospheric and high pressure.

Table II. Avrami exponent $n$

\begin{tabular}{|c|c|c|c|c|c|c|c|c|c|}
\hline \multicolumn{5}{|c|}{$M_{v}=15500$} & \multicolumn{5}{|c|}{$M_{v}=150000$} \\
\hline \multicolumn{2}{|c|}{$P=2490 \mathrm{~kg} / \mathrm{cm}^{2}$} & \multicolumn{3}{|c|}{$P=3000 \mathrm{~kg} / \mathrm{cm}^{2}$} & \multicolumn{2}{|c|}{$P=2340 \mathrm{~kg} / \mathrm{cm}^{2}$} & \multicolumn{3}{|c|}{$P=2490 \mathrm{~kg} / \mathrm{cm}^{2}$} \\
\hline$T,{ }^{\circ} \mathrm{C}$ & $n(\mathrm{~F})^{\mathrm{a}}$ & $T,{ }^{\circ} \mathrm{C}$ & $n(\mathrm{~F})$ & $n(\mathrm{E})^{\mathbf{b}}$ & $T,{ }^{\circ} \mathrm{C}$ & $n(\mathrm{~F})$ & $T,{ }^{\circ} \mathrm{C}$ & $n(\mathrm{~F})$ & $n(\mathrm{E})$ \\
\hline 186.6 & 2.9 & 196.6 & 2.8 & - & 183.6 & 3.3 & 187.8 & 3.3 & - \\
\hline 187.5 & 2.9 & 197.8 & 2.8 & 1.8 & 185.3 & 3.0 & 189.5 & 3.4 & 2.3 \\
\hline \multirow[t]{2}{*}{188.5} & 3.2 & 198.8 & 2.7 & 2.1 & 186.3 & 3.2 & 190.5 & 3.1 & 2.1 \\
\hline & & & & & 187.3 & 3.3 & 191.4 & 3.2 & 2.0 \\
\hline
\end{tabular}

${ }^{a} n(\mathrm{~F})$ denotes Avrami exponent of folded-chain crystallization.

${ }^{\mathrm{b}} n(\mathrm{E})$ denotes Avrami exponent of extended-chain crystallization. 


\section{Crystallization Rates}

Crystallization rates estimated from eq 2 are plotted against $T_{\mathrm{m}}{ }^{0} / T \Delta T$ in Figures 6 and 7 , where $T$ is the crystallization temperature, and $\Delta T$ is the degree of supercooling. First, the crystallization rates of the folded-chain crystals apparently become rapid with increasing pressure irrespective of the molecular weight. This result coincides with the studies of Kyotani and Kanetsuna, ${ }^{7}$ but conflict with the data of Calvert and Uhlmann who concluded that crystallization rates under high pressure were of the same order of magnitude as those at atmospheric pressure. ${ }^{1}$ Secondly, although direct comparison of the dependence of crystallization rates on pressure could not be made in the case of extended-chain crystallization, the crystallization rates of extended-chain crystals are considered to increase as pressure is increased. This is based on the consideration that the crystallization rate for a molecular weight of 15500 at $P=3000 \mathrm{~kg} / \mathrm{cm}^{2}$ is more rapid than that for a molecular weight of 150000 at $P=2490 \mathrm{~kg} / \mathrm{cm}^{2}$ as shown in Figure 7, in spite of the fact that the production of extended-chain crystals for a molecular weight of 15500 was more difficult than that for a molecular weight of 150000 at the same pressure. Thirdly, the extended-chain crystallization for a molecular weight of 150000 seems to show a maximum in the crystallization rate at the temperature not far from the melting temperature.

We suggest a schematic form of the dependence of the crystallization rate for both types of crystals upon the crystallization temperature as shown in Figure 8. It is estimated that the range of temperature in which both folded-chain and extended-chain crystallization can coexist is a few degrees. In regard to the first feature, we discuss the reason why the crystallization rate of folded-chain crystals increases as the pressure is increased.

The crystallization rate constant $k$ is controlled by the product $N G^{m}$, where $N$ is the nucleation frequency, $G$ the growth rate, and $m$ the integer depending on the mode of crystal growth. ${ }^{13}$ In the case of folded-chain crystallization, we assume that the theory of crystal growth is applicable to the case of crystallization under high pressure, and is described as

$$
G=G_{0} \exp \left(-\frac{U}{k T}\right) \exp \left(\frac{-\Delta F^{*}}{k T}\right)
$$

where $G_{0}$ is constant, $U$, the free energy of activation for transport across the crystal-liquid interface, and $\Delta F^{*}$, the activation free energy of the nucleus formation. ${ }^{14}$ In the case of two-dimensional growth, $\Delta F^{*}$ is expressed as $4 b T_{\mathrm{m}}{ }^{0} \sigma \sigma_{\mathrm{e}} / \Delta h \Delta T$, where $b$ is the molecular width, $\sigma$ the excess free energy of side chain, $\sigma_{\mathrm{e}}$ the excess free energy of the fold surface, and $\Delta h$, the heat of fusion.

Since the contribution of the term of $\Delta F^{*}$ to crystallization rate is assumed to be considerably larger near the melting temperature, $k$ is described as

$$
\log k=\text { const }-\frac{8 b \sigma \sigma_{\mathrm{e}}}{2.3 k \Delta h} \frac{T_{\mathrm{m}}{ }^{0}}{T \Delta T}
$$

Therefore, a plot of $\log k$ against the temperature variable $T_{\mathrm{m}}{ }^{0} / T \Delta T$ can give the estimation of $\sigma \sigma_{\mathrm{e}} / \Delta h$.

From Figure 6 it is shown that the value of $\sigma \sigma_{\mathrm{e}} / \Delta h$ decreased with increasing pressure up to the pressure studied. Therefore, under the condition of constancy in $T_{\mathrm{m}}{ }^{0} / T \Delta T$, the decrease in $\sigma \sigma_{\mathrm{e}} / \Delta h$ results in an increase in the crystallization rate under pressure through eq 4 . The values of $\Delta h$ and $\sigma$ are considered to vary a little with pressure, so that $\sigma_{\mathrm{e}}$ can be assumed to be dependent mostly on pressure, and thus decreases with increasing pressure.

At this stage, however, it is not certain why the value of $\sigma_{\mathrm{e}}$ decreases as the pressure is increased. The decrease in the value of $\sigma_{\mathrm{e}}$ may be related to the decrease in the number of gauch conformations in the fold suface with pressure.

We now estimate the lamellar thickness of foldedchain crystals produced under high pressure. Even if within ten degrees of supercooling, it was possible to cause folded-chain crystallization. Since the lamellar thickness of folded-chain crystals is proportional to the inverse of supercooling, the lamella is expected to be thick compared with that for atmospheric pressure crystallization which generally necessitates more than ten degrees of supercooling.

Usually, the lamellar thickness $l$ is expressed as

$$
l=\frac{2 \sigma_{\mathrm{e}}}{\Delta h} \cdot \frac{T_{\mathrm{m}}{ }^{0}}{\Delta T}
$$

Thus, the ratio of lamellar thickness at pressure $p$ to that at atmospheric pressure is described as

$$
\frac{l_{p}}{l_{1}}=\frac{\left(T_{\mathrm{m}}{ }^{0} / \Delta T\right)_{p}}{\left(T_{\mathrm{m}}{ }^{0} / \Delta T\right)_{1}} \frac{\left(\sigma_{\mathrm{e}} / \Delta h\right)_{p}}{\left(\sigma_{\mathrm{e}} / \Delta h\right)_{1}}
$$




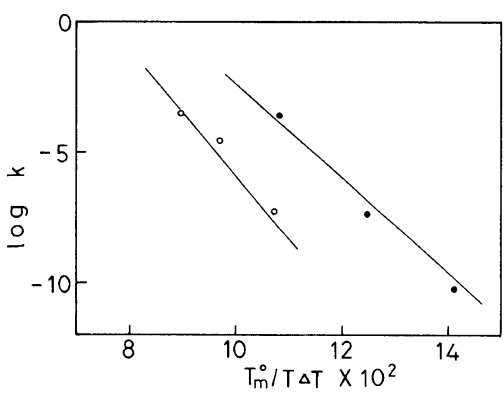

(a)

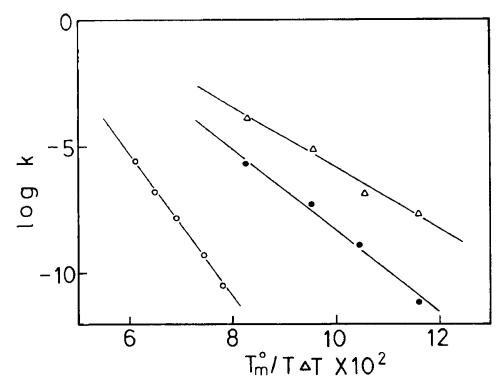

Figure 6. Plot of the crystallization rate constant $k$ of folded-chain crystals against $T_{\mathrm{m}}{ }^{0} / T \Delta T$ : (a) pressure at (○) $2490 \mathrm{~kg} / \mathrm{cm}^{2}\left(T_{\mathrm{m}}{ }^{0}=197.9^{\circ} \mathrm{C}\right)$ and (O) $3000 \mathrm{~kg} / \mathrm{cm}^{2}$ $\left(T_{\mathrm{m}}{ }^{0}=206.1^{\circ} \mathrm{C}\right)$ for $M_{v}=15500 ;(\mathrm{b})$ pressure at $(\mathrm{O})$ atmospheric pressure $\left(T_{\mathrm{m}}{ }^{0}=143^{\circ} \mathrm{C}\right)$, data of ref $12,(\mathrm{O})$ $2340 \mathrm{~kg} / \mathrm{cm}^{2}\left(T_{\mathrm{m}}{ }^{0}=196.2^{\circ} \mathrm{C}\right)$, and $(\triangle) 2490 \mathrm{~kg} / \mathrm{cm}^{2}$ $\left(T_{\mathrm{m}}{ }^{0}=200.1^{\circ} \mathrm{C}\right)$ for $M_{v}=150000$.

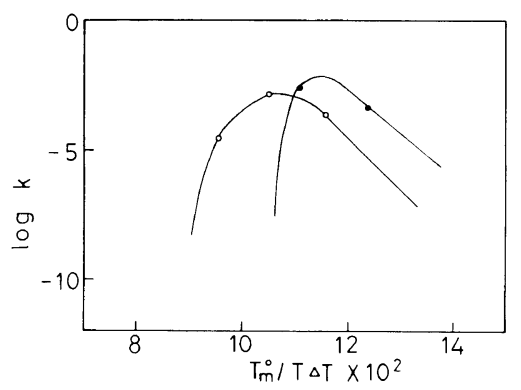

Figure 7. Plot of the crystallization rate constant $k$ of extended-chain crystals against $T_{\mathrm{m}}{ }^{0} / T \Delta T$ : $M_{v}=15500$ and (O) $M_{v}=150000$.

As an example of lamellar thickness at high pressure, let's take a case of folded-chain crystallization at $T=191.4^{\circ} \mathrm{C}$ and $P=2490 \mathrm{~kg} / \mathrm{cm}^{2}$ for a molecular weight of 150000 . As a temperature variable at atmospheric pressure, a variable which gives the same crystallization rate as that for a

Polymer J., Vol. 11, No. 6, 1979

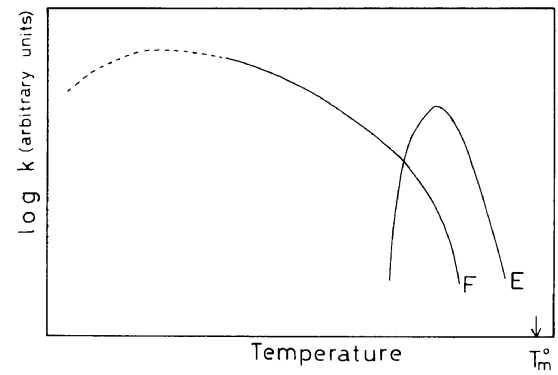

Figure 8. Schematic diagram of crystallization rates for both types of crystals. F denotes folded-chain crystallization and $\mathrm{E}$ denotes extended-chain crystallization.

molecular weight of 150000 may be chosen, and in this case $T=128^{\circ} \mathrm{C}$ was used. With this temperature variable, the calculation that $\left(T_{\mathrm{m}}{ }^{0} / \Delta T\right)_{p} \simeq 2.0$ $\left(T_{\mathrm{m}}{ }^{\mathrm{O}} / \Delta T\right)_{1}$ could be made.

On the other hand, from Figure 6a it is evaluated that $\left(\sigma_{\mathrm{e}} / \Delta h\right)_{p} \simeq 0.45\left(\sigma_{\mathrm{e}} / \Delta h\right)_{1}$ if the dependence of $\sigma$ on pressure is ignored. Therefore, it can be considered that $l_{p}$ is almost identical to $l_{1}$. Under the condition of the same crystallization rate, the lamellar thickness of folded-chain crystals is considered unaffected by pressure.

\section{REFERENCES}

1. P. D. Calvert and D. R. Uhlmann, J. Polym. Sci., Part A-2, 10, 1811 (1972).

2. H. Kanetsuna, S. Mitsuhashi, M. Iguchi, T. Hatakeyama, M. Kyotani, and Y. Maeda, J. Polym. Sci., Part C, 42, 783 (1973).

3. M. Yasuniwa, C. Nakafuku, and T. Takemura, Polym. J., 4, 526 (1973).

4. D. C. Bassett and B. Turner, Phil. Mag., 29, 285 (1974).

5. D. C. Bassett and B. Turner, Phil. Mag., 29, 925 (1974).

6. M. Yasuniwa, R. Enoshita, and T. Takemura, Jpn. J. Appl. Phys., 15, 1421 (1976).

7. M. Kyotani and H. Kanetsuna, J. Polym. Sci., Part A2, 12, 2331 (1974).

8. T. Hatakeyama, H. Kanetsuna, H. Kaneda, and T. Hashimoto, J. Macromol. Sci., B 10, 359 (1974).

9. Y. Maeda and H. Kanetsuna, J. Polym. Sci., Part A-2, 14, 2057 (1976).

10. P. J. Flory, J. Am. Chem. Soc., 84, 2857 (1962).

11. H. H. Hoehn, R. C. Ferguson, and R. R. Hebert, Polym. Eng. and Sci., 18, 457 (1978).

12. S. Sawada and T. Nose, Polym. J., 11, 227 (1979).

13. L. Mandelkern, "Crystallization of Polymers," McGraw-Hill, New York, N.Y., 1964, Chapter 8.

14. D. Turnbull and J. C. Fischer, J. Chem. Phys., 17, 71 (1949). 